\title{
Archéologie du politique chez François Bon
}

\author{
Stéphane Inkel \\ Université Queen's
}

C'est maintenant une idée largement reçue d'affirmer l'inaccessibilité du réel, le XXe siècle ayant confirmé la justesse de la théorie lacanienne d'une détermination du sujet par un Réel qui échappe pourtant à la nomination (Symbolique), comme Slavoj Žižek en a fait la démonstration en déplaçant la thèse d'Alain Badiou voulant que la question majeure du siècle ait été la "passion du réel » (dans sa volonté «d'atteindre à la chose elle-même» [p. 24]) puisque celle-ci ne manque pas de verser dans son contraire, «le pur semblant de l'effet de réel spectaculaire » (défilés staliniens, actes terroristes, etc.). L'une des illustrations les plus éclatantes de cette inaccessibilité, 
pourtant riche d'effets multiples et concrets, réside dans l'analyse du système financier ultra libéralisé ayant mené à la crise de 2008 faite par Žižek. Commentant la «critique de l'économie politique» du dernier Marx, l'auteur évoque le caractère réel de la «danse spéculative» du Capital dans la mesure où cette "autofécondation", abstraite, "objective et anonyme » (Žižek, 2008, p. 27-28), n'entraîne pas moins des effets très concrets :

Nous rencontrons ici la différence lacanienne entre la réalité et le Réel : la "réalité" désigne la réalité sociale dans laquelle des gens réels interagissent dans le cadre du processus productif matériel; le Réel, lui, désigne l'inexorable logique, spectrale et "abstraite", qui détermine la scène de la réalité sociale. (p. 28)

Il revient toutefois à François Bon d'avoir montré que le Réel le plus reculé est politique au moins autant qu'économique. C'està-dire que le politique, en tant qu'espace délibératif fait de tensions et de rapports de force où ceux qui sont «sans part» parviennent à se faire entendre, suivant la définition de la démocratie en tant que mésentente de Jacques Rancière, devient de plus en plus inassignable et d'autant plus lourd d'effets. On sait que si les préoccupations politiques n'étaient pas absentes de Sortie d'usine, elles étaient encore classiquement organisées par le registre de l'aliénation des travailleurs, mis à distance par un formalisme relativement appuyé. Dès le livre Impatience s'exprime toutefois un désir pour le «pur documentaire » (Bon, 1998, p. 12) fondé sur l'inventaire exhaustif à la fois des lieux et des histoires des hommes et des femmes qui les habitent, désir qui, après la publication du rendu de l'expérience des premiers "ateliers d'écriture», notamment Prison, atteint toute son efficace dans Daewoo en 2004. Ce retour du réel, par la brèche du «documentaire», semble donc opérer sur deux plans, 
esthétique et politique. Car il en va d'abord du genre "roman » à travestir, par le recours à des formes extrêmement hétérogènes, les extraits de journaux à la langue de bois des politiques, en passant par la parole prêtée aux ouvrières, aux extraits de la pièce de théâtre que Daewoo a d'abord été, sans parler de la photographie que Bon évoque ici avec régularité et qui occupe une place centrale dans d'autres récits de l'écrivain, de Paysage Fer à Billancourt, consacré au même environnement industriel (voir Jérusalem, 2010). Il faut aussi ajouter la fascination de l'écrivain pour la géométrie des lieux, des surfaces planes vidées de leur équipement des usines à la dernière lettre, toute perecquienne - faisant signe vers un certain formalisme - de la raison sociale de l'usine se baladant dans le ciel ${ }^{1}$. François Bon a déjà dit, dans un entretien, le lien d'abord esthétique qui le lie au paysage industriel des aciéries, où il a travaillé en tant qu'ingénieur avant son passage à l'écriture : «Un grand choc pour moi a été ma première saison dans les aciéries de Longwy, vers 1974. C'était beau et puissant. Immensément beau dans son pragmatisme d'architectures superposées [...]. Quinze ans plus tard, c'est toujours vers cette esthétique que j'avance [...]. »² Dans un premier temps, il semble donc que les données du rapport au politique soient renversées et que ce soit le réel qui vienne en aide à l'écrivain

\footnotetext{
1 « La disparition progressive des six lettres, d'abord comme on efface à la machine, enlevant les dernières lettres. Quand j'étais arrivé, c'est un 0 majuscule qui se promenait dans le ciel, soulevé par le bras jaune de la grue au-dessus du rectangle bleu de l'usine : et DAEWO puis DAEW puis AEW puis EW, enfin ce seul W au lieu de DAEWOO, écrit en géant sur l'usine. » (Bon, 2006, p. 77)

2 « En marge », entretien avec Jean-Christophe Millois, Prétexte, 1995, cité par Vray, 2010, p. 138.
} 
dans sa refonte de la forme " roman ». Pourtant, c'est à travers une telle redéfinition qu'il est loisible de repérer le rapport au politique entretenu par le roman. D’une part, la prémisse de ce livre concerne ce réel lui-même dans ce qu'il a d'à la fois inatteignable et irréductible, soit la volonté d'incarner la statistique du chômage à la suite de la fermeture des trois usines du groupe coréen dans la vallée de la Fensch. Un volet important du livre est donc consacré à «l'enquête » effectuée par le narrateur / écrivain sur les lieux désaffectés (« ne rien présenter que l'enquête » [Bon, 2006, p. 247]), compilation d'un dossier sur «l'affaire» et, surtout, archivage de la parole, ignorée, des ouvrières. Mais d'autre part, et au-delà de cette posture de témoin, c'est dans la définition même de son projet d'écriture que l'écrivain peut nous aider à repenser, méthodologiquement, les rapports entre politique, écriture et espace critique: "Mon travail, c'est de rendre compte par l'écriture de rapports et d'événements qui concernent les hommes entre eux. » (Bon, 2006, p. 190) Comment traduire ce rapport entre les hommes, ou plutôt l'absence de rapport entre des travailleurs, à majorité des femmes, privés du travail de l'usine, et des interlocuteurs - économiques et politiques qui font défaut? C'est donc à la mise en scène de ces microrelations en tant que figurations $\mathrm{du}$ politique à l'état embryonnaire que je m'intéresserai, cherchant à repérer dans cette recension des rapports intersubjectifs une archéologie $d u$ politique qui se confond avec la nature même du récit plutôt que d'en être simplement l'objet. 


\section{Un souci du monde malgré tout}

Distinguant la littérature contemporaine, dont ils proposent de situer l'origine en 1980, de celle, formaliste, des décennies antérieures, «peu à peu persuadée qu'elle ne pouvait échapper à la clôture du langage » (2008, p. 15-16), Dominique Viart et Bruno Vercier font du retour de l'objet l'une de ses caractéristiques, proposant de parler d'une littérature «transitive ». Il y a toutefois quelque chose de paradoxal à apparenter ainsi les expériences formalistes à un déni du monde, alors même que l'engagement marxiste de toute une génération atteignait un sommet à la suite de Mai 68, accompagné par les figures éminentes de ce même formalisme, Sollers en tête. S'il faut opposer cette littérature " intransitive » à la fois à celle qui lui succède et à celle de ses devanciers plus directement "engagés », de Sartre à Aragon, il faut donc aussi en rappeler la radicalité politique - pensons au manifeste « La révolution ici maintenant ${ }^{3}$ » publié par Tel Quel (no 34, octobre 1968) - qu'elle situait simplement en amont de l'objet, dans la structure langagière du sujet et son rapport à l'Autre. Voyons de plus près.

Dans sa biographie de James Joyce, Richard Ellmann raconte comment le jeune Beckett passait souvent des heures

\footnotetext{
3 Manifeste signé par l'ensemble du comité de rédaction de l'époque, de Sollers, Kristeva et Pleynet à Hubert Damish, Pierre Boulez ou Jean-Louis Shefer. Rappelons l'une de ses thèses, qui pour s'appuyer sur la «théorie du langage » au fondement de l'entreprise, s'inscrit dans un mouvement plus large de mise à nu des idéologies présentes dans le discours social de l'époque : « 5. La construction, par conséquent, d'une théorie tirée de la pratique textuelle que nous avons à développer nous semble susceptible d'éviter les impasses répétitives du discours "engagé" - modèle d'une mystification téléologico-transcendantale humaniste et psychologiste, complice de l'obscurantisme définitif de l'état bourgeois ; » Texte en

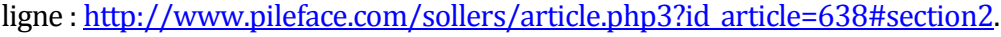


en compagnie de son aîné, poussant le mimétisme envers le maître jusqu'à porter les mêmes petites lunettes rondes et à plier la jambe de la même façon lors de leurs tête-à-tête silencieux. Car tous deux aimaient le silence, qu'ils pouvaient conserver pendant des heures, à la différence près, précise Ellmann, que Beckett songeait surtout au monde, alors que Joyce songeait d'abord à lui-même (1987, p. 297).

Cette anecdote sous forme de boutade a le mérite de nous rappeler cette évidence largement passée sous silence par les catégories un peu simples que nous utilisons lorsqu'il s'agit de classer ou simplement décrire une œuvre : une littérature qui se détourne du monde ne signifie pas qu'elle s'en désintéresse.

Pourtant, nulle œuvre, on le sait, ne s'est autant détournée du monde, de l'objet, que l'œuvre de Beckett, que ce soit dans son théâtre, illustration d'une panne de toute événementialité, ou dans ses récits, où il ne s'agit plus de raconter quoi que ce soit, mais bien d'énoncer les conditions de la voix qui se fait entendre. Sans doute, on ne ferait guère de cas de l'anecdote si ce "souci du monde» ne trouvait son exact équivalent dans une œuvre aussi tardive que Compagnie :

Assommé par les maux de ton espèce tu soulèves néanmoins la tête de l'appui des mains et rouvres les yeux. Tu branches sans bouger de ta place la lumière au-dessus de ta tête. Tes yeux tombent sur la montre sous tes yeux. Mais au lieu de relever l'heure de la nuit ils suivent les girations de la trotteuse que son ombre tantôt précède et tantôt suit [...]. Mais n'en pouvant plus tu laisses retomber la tête là où elle était et les yeux fermés retournes aux maux de ton espèce (Beckett, 1993, p. 41-43. Je souligne).

Ce qu'il s'agirait de penser, je ne l'indique ici qu'à titre d'hypothèse, c'est qu'un tel repli de l'énonciation sur elle-même 
n'est pas tant une fuite du monde qu'une autre forme de figuration du politique par le roman. Je m'explique.

Dans l'une de ses Vies politiques consacrée à Lessing, Arendt a qualifié d'«émigration intérieure» (1986, p. 32) le repli sur soi du sujet quittant la pluralité du monde pour la sphère privée, attitude s'accompagnant d'une "perte en monde» (p.13). Il est toutefois certaines époques où cette « émigration intérieure » devient résistance, regard critique sur les « sombres temps » dont il se retire. La qualité politique d'un tel sujet ne se mesure donc pas à son action - ce qu'Arendt appelle "action», qui peut se résumer à une prise de parole initiante sur la scène délibérative - , mais à ce quelque chose d'indéfinissable que constitue sa préoccupation, forme d'«oasis», pour reprendre l'une de ses métaphores les plus courantes, dans le « désert du monde » (Arendt, 2001, p. 187).

L'œuvre de Beckett et, avec lui, celle d'une partie importante de ses contemporains peuvent être qualifiées d' " émigration intérieure ». Aux antipodes de tout engagement, elles offrent pourtant cette chance de figurer quelque chose, endeçà de toute idéologie, de l'ordre des conditions du politique, soit des micro-relations de pouvoir s'exerçant dans la rencontre du sujet et de l'Autre - que cet Autre représente la «langue de la tribu », comme dans L'Innommable, ou plus simplement autrui comme dans Comment c'est - et de tout un travail de la part du sujet en vue de l'établissement d'une distance nécessaire entre lui et cet Autre, travail de langue en vue de rompre le dispositif de sa propre subjectivation (voir Agamben, 2007).

Si l'œuvre de François Bon est particulièrement exemplaire dans son rapport au politique, c'est qu'elle réunit un 
héritage formaliste aisément identifiable, dans sa syntaxe comme dans sa méfiance à l'égard des dispositifs de la subjectivité, de même qu'une préoccupation «transitive » pour le réel repérable aussi bien dans ses ateliers d'écriture que par son usage à tous égards novateur des possibilités du web ${ }^{4}$. Ce retour au réel ne s'est toutefois pas effectué d'un seul tenant. Entre ces deux romans sur l'usine que sont Sortie d'usine et Daewoo, c'est une profonde mutation de la manière de problématiser le politique qui se fait jour. Or, c'est dans ce dernier, au matériau fortement hétérogène, comme je le disais plus tôt, donc, particulièrement ouvert sur le dehors, qu'il semble possible d'esquisser un parallèle avec la leçon archéologique de Beckett, tant ce qui est en jeu concerne l'établissement d'une mise en relation d'une parole, dans un premier temps ignorée et condamnée à tourner à vide, et d'un Autre que le roman s'emploie à construire. C'est donc à cette mutation qu'il s'agit d'être attentif, à un changement de paradigme qui concernerait aussi bien ce monde avec lequel l'œuvre dialogue que sa propre manière d'envisager cette relation.

\section{De Sortie d'usine à Daewoo, mutation du politique}

Jacques Rancière, dans sa contribution à l'exploration actuelle de L'Idée de communisme proposée par Alain Badiou et Slavoj Žižek intitulée "Communistes sans communisme », formule, à partir de la pensée de l'émancipation de Joseph Jacotot,

\footnotetext{
4 Bon est notamment l'instigateur de l'excellent site remue.net sur les écrivains contemporains, de l'éditeur numérique publie.net, sans parler de son site personnel d'expérimentations, tierslivre.net.
} 
pédagogue des Lumières, l'idée d'un «communisme de l'intelligence » (2010, p. 233) qui se veut la réponse au principe platonicien de l'aptitude de l'artisan à l'exercice de son travail (et donc de son inaptitude pour toute autre fonction). Dans cette démonstration de la " capacité des incapables », il s'agirait de faire en sorte de souligner «la capacité pour l'ouvrier de laisser son regard et son esprit s'évader du travail de ses mains [et] de transformer l'espace privé de l'atelier en espace public » (ibid.). Ce qui serait en jeu, dans ce nouveau «partage du sensible ", c'est le refus de la minorisation des travailleurs qu'implique l'établissement d'un temps organisé qui élimine les conditions d'un libre exercice de la pensée; le refus, également, de la ligne de partage qui sépare l'ouvrier et son travail du décideur (actionnaires et gouvernants) qui en dispose les conditions. On trouve dans Daewoo un récit qui fait écho à un tel bouleversement des places ou, du moins, qui propose la subversion de cette aptitude qui exclut de l'ouvrier. Dans cette description de Sylvia F., figure centrale du soulèvement des ouvrières de l'usine menacée de fermeture, rapportée au narrateur par une ancienne compagne de travail à la suite de son suicide, on voit à l'œuvre un comportement en vérité tout simple, qui a toutefois pour vertu d'ouvrir un imaginaire qui se superpose aux gestes qu'il accompagne et qui les rectifie :

Sylvia, c'était celle qui nous inventait les fables [...]. La chef pouvait rien dire : on regardait devant nous, on ne la regardait même pas, quand elle nous disait son histoire [...]. Ce n'était pas à voix forte ou haute, non, le bruit de l'usine plutôt qu'on le tenait à distance: le chuchotement de Sylvia nous on l'entendait, c'est à nous qu'elle parlait, tandis qu'on continuait les gestes. (Bon, 2006, p. 24)

Malgré le caractère modeste d'une telle faculté de fabuler sur la chaîne de montage, il faut toutefois apprécier l'écart qui sépare 
cette seule possibilité de la réalité du travail décrit vingt ans plutôt dans Sortie d'usine, où la productivité se mesure au contraire par la capacité de la chaîne de montage d'« engourdir» la pensée. Car ce qu'il faut remarquer, c'est que, dans cet exemple, c'est bien l'usine qui permet de rêver à la suite, alors que sa fermeture va entraîner une perte qui laisse certaines ouvrières dans le désarroi du désœuvrement. On verra aussi qu'un rapport de force proprement politique ne peut exister qu'à partir de l'usine, sans quoi leur parole ne dispose plus d'aucune place. C'est donc vers l'apparition d'un véritable « litige politique » que le roman nous entraîne, au sens que Rancière donne à ce terme en tant qu'il «n'est pas une discussion entre partenaires mais une interlocution qui met en jeu la situation même d'interlocution» (1995, p.140-141). Entre les deux expériences, c'est donc le statut de l'aliénation de l'ouvrier qui se trouve rectifié, voire la conception du sujet, qui en retour modifie la nature de la confrontation politique. Il est donc remarquable de voir ainsi un écrivain témoigner à travers son œuvre d'un changement de paradigme majeur, le roman accompagnant à même ses choix de représentation une modification du discours tenu sur le politique.

Car à sa manière, Sortie d'usine constitue une forme d'achèvement, point culminant d'une description de l'expérience ouvrière sous le signe de son aliénation. Pour le dire rapidement, ce roman a fait date en raison de sa réussite à rendre compte d'une sorte de " conscience de l'usine » à partir de l'expérience vécue de Bon lui-même, chez qui, phénomène rare, cette expérience de l'usine précède celle de l'écriture ou d'une appréhension proprement politique de sa réalité, comme ce pouvait être le cas chez les militants de certains groupes marxistes ou maoïstes « établis » en usine pour y faire circuler 
une analyse préétablie. Comme le dit avec force Pierre Bergounioux, « ce qui est neuf, c'est qu'on voit [l'usine] avec les yeux de ceux qui, pour lui être immédiatement affrontés, ne peuvent pas l'envisager; c'est que, pour la première fois, une voix s'élève du dedans pour dire au lecteur, au monde du dehors, ce qui se passe dedans. » $(1995$, p. 106) Le secret d'un tel dévoilement, aux yeux de la lecture très bourdieusienne de Bergounioux, réside dans l'« hétérogamie » de l'écrivain, c'est-àdire de la confrontation de l'hérédité ouvrière de la lignée paternelle et d'un héritage littéraire du côté maternel qui l'a conduit à se doter très jeune d'une culture qui l'aidera plus tard à considérer l'usine d'un autre regard 5 . Il invente donc le langage, à la fois fortement stylisé, formaliste et impersonnel, lui permettant de décrire, c'est-à-dire nommer, ce qui concourt à l'aliénation de l'ouvrier et qui le dépossède jusqu'à le priver de l'usage de la première personne : temps fortement organisé, fatigue, répétition. L'ouverture du roman est à cet égard exemplaire. En effet, celle-ci donne à voir un lieu de transit vers l'usine : une gare — «laquelle n'importe » (Bon, 1982, p. 7) — à partir du point de vue du sujet anonyme sur lequel se concentre la focalisation - sorte d' «everyman » désubjectivé par l'usage appuyé de l'infinitif qui sert ici d'impératif impersonnel. On le trouve donc aux prises avec la contrainte du temps plein, précis, de l'usine (pointer à temps, être à sa place lorsque les machines sont mises en branle, etc.), dont il offre une description des plus évocatrices: "Le matin était entamé déjà [...] une fois la première heure faite, celle de mise en route, puis les deux

5 Comme l'écrit Bergounioux avec un art de la synthèse et de l'exagération dont il a le secret, " l'œuvre de François Bon est écrite. Il ne lui reste qu'à la porter sur le papier. » (Bergounioux, 2010, p. 147) 
suivantes qui sont un peu le plein de la journée, où se superpos[e] l'énergie encore du début à l'engourdissement pas dissipé tout à fait de la pensée [...].» (p.33. Je souligne) Le roman s'attarde ainsi longuement sur « la succession rigide des actes, leur enchaînement mécanisé » (p.34) et sur les divers événements qui l'interrompent (accidents de travail, grève) et permettent de le saisir. L'usine y est donc dépeinte de l'intérieur par un regard enclos, mais qui, pour une raison ou une autre, s'en est échappé et peut en rendre compte. Car grâce à une «distance » soudainement "définitivement acquise » (p. 152), cet anonyme que l'on a suivi tout au long du roman sans l'entendre quitte l'usine et assume à ce moment très précis la narration homodiégétique du texte: «[L'usine] ne m'était visible que parce que je m'en étais sorti, plus que dehors ${ }^{6} . »$ (p. 161)

Cette menace qui pèse sur la subjectivité en raison du temps plein de l'usine qui limite l'exercice de la pensée est précisément ce qui a disparu de Daewoo, où l'usine est plutôt décrite comme étant l'adjuvant nécessaire à la parole, à la faculté de rêver, à la vie même, si l'on suit scrupuleusement la logique derrière le suicide de Sylvia F., celle-là même qui raconte les histoires pendant le travail sur la chaîne de montage. Comment interpréter un tel déplacement? Et surtout, en quoi les coordonnées du politique s'en trouvent-elles bouleversées?

\footnotetext{
${ }^{6}$ La chance de Bon et de son héritage littéraire lui ayant permis de quitter une réalité qui aurait pu être aliénante est également prêtée au narrateur qui, à la fin du roman, reconnaît sa dette au Château de Kakfa : "Si j'avais pu quitter l'usine, échapper d'où l'usine tolère et enclôt soigneusement en elle ce qui en elle s'affronte à ses lois, c'est d'avoir autrefois, aveuglément, vécu l'usine comme si j'en étais encore à lire Kafka. » (p. 165)
} 


\section{Le mécompte des « invendables »}

Prolongeant l'ouverture du roman - «Refuser. Faire face à l'effacement même.» (Bon, 2006, p. 9) -, de nombreux critiques mettent au centre de leur lecture cette lutte contre l'effacement, que ce soit de la réalité du chômage, souvent limitée à sa statistique et progressivement acceptée comme faisant partie du visage économique de l'époque, ou des vestiges de l'ère industrielle de la vallée de la Fensch avec les nombreux hauts fourneaux de ses aciéries (voir van Montfrans). Mais l'effacement se limite-t-il vraiment à cette réalité visible? Daewoo n'est ni un roman régionaliste (qui défendrait par exemple la spécificité socio-économique d'une région), ni un roman " engagé » à proprement parler, même si le choix de se tenir du "côté des vaincus » (p. 9) est énoncé dès la première page. Ce qui est refusé, en premier lieu, c'est une certaine normalisation de la crise engendrée par la fermeture des trois usines du groupe coréen. C'est le fait «que tout ici, en apparence, continuait comme avant» (p.11) qui se voit questionné, le cours «normal» du devenir qui violente et appelle un retour documentaire pour ainsi dire "géologique » sur l'événement en amont: le roman multipliera ainsi les couches discursives de manière à saturer l'événement luimême, à le faire occuper tout l'espace de la narration par le matériau de l'enquête qui agit comme une sorte d'arrêt sur image sur les vies qui s'en trouvèrent bouleversées. C'est d'ailleurs ce qui retient d'emblée notre attention à la lecture, cette intrication inusitée entre les formes de l'enquête, voire les outils du documentaire, et du langage fictionnel lui-même très hétérogène (théâtral, métadiscursif, etc.). 
C'est donc à cette intrication particulière qu'il faut être attentif, notamment en s'imposant comme première tâche méthodologique de dénouer l'ambiguïté soigneusement entretenue par Bon entre la figure de l'auteur et le narrateur du roman par les nombreuses indications biographiques et événementielles vérifiables dans les limites du livre. Car on cite volontiers la dernière phrase du roman, « ne rien présenter que l'enquête» (p. 247), sans nécessairement en soupeser pleinement les implications. L'opération est d'autant plus importante que le roman se propose de réaliser essentiellement deux tâches à travers l'enquête : 1 . Restituer une voix, ou plutôt un groupe de voix demeurées inaudibles au cœur des événements qui ont entouré la fermeture des trois usines; 2. distinguer l'écheveau des rapports, par définition politiques, dans lesquelles ces voix retentissent ou auraient dû retentir : à qui elles s'adressent, ce qu'on leur répond, de quel lieu et avec quel typologie discursive. C'est à travers la première tâche que l'on fait face à un double écueil. Il faut d'abord départager le vrai du faux derrière la prétention maintes fois répétée d'un effacement du narrateur face aux discours des ouvrières prétendument enregistrés sur «mini-disc» au cours d'entretiens. Le narrateur s'explique d'ailleurs à de nombreuses reprises sur la nécessité de transposer plutôt que de transcrire la parole recueillie, la mimesis devenant ici le garant d'une plus grande authenticité dans la mesure où l'écriture permettrait d'aller au-delà du seul « dit » des entretiens :

Je ne prétends pas rapporter les mots tels qu'ils m'ont été dits : j'en ai les transcriptions dans mon ordinateur, cela passe mal, ne transporte rien de ce que nous entendions, mes interlocutrices et moi-même, dans l'évidence de la rencontre [...]. C'est cela qu'il faut reconstruire, seul, dans les mois qui suivent, écoutant une fois de plus la voix, se remémorant ce 
qu'on apercevait de la fenêtre, comme les noms et prénoms cités [...]. J'appelle ce livre roman d'en tenter la restitution par l'écriture, en essayant que les mots redisent aussi ces silences, les yeux qui vous regardent ou se détournent, le bruit de la ville tel qu'il vous parvient par la fenêtre [...]. (p. 42)

Maintenant que nous savons que ces entretiens ont été inventés par l'écrivain7, le souci d'exactitude et le scrupule du narrateur allant jusqu'à prévenir son lecteur des ajouts apportés à la transcription afin de rester au plus près de la vérité de l'instant prend évidemment un tout autre sens. C'était là le second écueil auquel nous confrontait cette restitution, d'avoir à reconduire les lieux communs habituels sur la capacité du roman à relayer une voix qui ne trouverait pas de lieu pour se faire entendre, faisant ainsi de l'écrivain le porte-parole d'une minorité muette. $\mathrm{Ne}$ pouvant atteindre directement le réel de l'événement, le récit se sert donc de la forme "enquête» pour revivifier son récit. En d'autres mots, le prétendu « réalisme » de l'enquête est précisément ce qui permet au roman de s'approcher de ce qui n'a jamais été visible dans la crise, puisque le litige n'a pu trouver l'espace de son avoir-lieu. Ainsi, Daewoo n'est peut-être pas tant un témoignage sur «l'affaire » qui lui donne son nom qu'un roman qui permet de saisir la nouvelle configuration dans laquelle interviennent-ou précisément ne peuvent intervenir - les différents acteurs sociaux dans l'espace politique propre à l'économie néo-libérale.

7 On trouve notamment cette information, confirmée maintes fois par l'écrivain, dans la note 5 d'un article de Christine Jérusalem: «Lors du colloque, François Bon révèlera au cours d'un entretien qu'il n'a pu rencontrer les ouvrières de Daewoo et que tous les entretiens sont inventés. » (2010, p. 181) 
C'est à ce titre que certains ont pu considérer que Daevoo était d'abord l'illustration d'une impasse. C'est notamment le point de vue de Stéphane Chaudier, qui voit dans le roman l'illustration des impasses de la pensée politique contemporaine, du moins dans son héritage marxiste : "Que reflète, dans Daewoo, ce jeu des impasses en miroir? [...] L'impasse sociale et politique des personnages en lutte accuse l'impasse d'une esthétique militante dépourvue, à cause du déficit de la pensée politique contemporaine, d'un imaginaire de l'action subversive et efficace.» (2010, p.172) Mais précisément, on peut ici le dire avec force, la fonction du roman n'est pas de représenter l'action, mais de circonscrire les conditions dans lesquelles elle pourrait s'exercer. C'est à cela que s'applique François Bon lorsqu'il définit l'écriture comme une mise en rapport d'événements et des "hommes entre eux » (2006, p. 190) ou comme la production de «liens » entre voix, visages et l'ensemble d'un territoire (p. 13). À cet égard, toute l'entreprise du roman vise à reproduire à l'échelle de la représentation ce qu'Hannah Arendt entend par "pluralité », cette qualité « d'entre-deux [qui] relie et sépare en même temps les hommes» (1994, p.92) et au sein de laquelle l'action authentiquement politique est avant tout un fait de parole agonistique qui apparaît entre les hommes (p. 240). C'est à ce titre que la liberté est à la fois initiante et " non souveraine ${ }^{8}$ ", puisqu'elle doit s'en remettre à la parole des autres qui lui font face et sans qui elle ne saurait exister. Ce lieu agonistique, on le sait, a fait défaut, et parce qu'il a fait défaut, une certaine violence a pu surgir et être retournée contre les ouvrières pour justifier, encore une fois a fortiori, l'absence de dialogue. Si

8 Sur cette notion de « liberté non souveraine », voir Villa (2008, p. 158-172). 
l'activité politique est ce qui « fait entendre comme discours ce qui n'était entendu que comme bruit » (Rancière, 1995, p. 53), on pourrait considérer que Daewoo en enregistre l'absence. À moins de considérer l'espace propre du roman comme le lieu où ce bruit devient discours.

Il existe donc un double diagnostic au sein du roman : 1. diagnostic proprement social, où il s'agit de rendre compte de la mutation de l'économie de toute une région et du désœuvrement que cette mutation engendre; 2 . diagnostic sur l'état du politique contemporain, où l'atomisation des lieux de pouvoir expulse les lieux du litige en dehors de la réalité sociale. Tout l'enjeu consiste à mettre en rapport cette double mutation qui affecte l'économie et le politique. Il appartient à Sylvia F., ce personnage fictif de suicidée qui est la condensation de trois suicides réels ayant suivi de peu la fermeture des usines, de formuler une approximation de cette mise en rapport telle qu'elle est rapportée par l'un des entretiens fictifs au début du roman : «Et à nous, au retour, ce truc bizarre, soi-disant qu'elle avait lu ça dans la Bible : - La sueur de ton front maintenant est invendable, quand tu mangeras ton pain tu sauras que la sueur de ton front est superflue. On est les superflues, elle avait ajouté. » (Bon, 2006, p. 25) Cette «sueur superflue»des invendables s'avère une métaphore particulièrement évocatrice des effets spécifiques de la mutation de l'univers du travail sur la durée-que résume à merveille une expression d'un fonctionnaire du gouvernement français, cité dans le roman à partir d'un article du journal Le Monde : « Le temps est à l'usine jetable.» (p. 93) Ce que le roman de François Bon permet de penser, à partir d'une telle expression, c'est que ce n'est pas seulement l'économie qui est délocalisée, mais bien le politique lui-même, fractionné et dépersonnalisé à l'extrême face aux 
diktats d'un marché irréductible et insaisissable. C'est la réponse à cette délocalisation qui me semble particulièrement intéressante dans ce roman. En restituant dans les limites du récit un rapport qui n'a pas eu lieu en superposant les discours des divers acteurs de la crise, il cherche à figurer les conditions originelles du politique, son archéologie, en esquissant le réseau complexe de voix qui ont à se heurter l'une l'autre pour donner lieu au « litige » proprement dit. L'éthique de son travail pourrait donc être définie minimalement comme le fait de donner à voir ces conditions élémentaires et d'en restituer l'un des acteurs, menacé d'oubli - à l'image du fantôme de Sylvia F., cet autre réel inatteignable, dont il ne reste rien que les suicides incompris qu'elle condense et dont l'écrivain refuse l'effacement définitif.

\section{Bibliographie}

Agamben, Giorgio. (2007), Qu'est-ce qu'un dispositif?, Paris, Rivages, coll. « Petite Bibliothèque ».

ARENDT, Hannah. (2001), Qu'est-ce que la politique?, texte établi par Ursula Ludz, traduction de l'allemand par Sylvie Courtine-Denamy, Paris, Seuil, coll. « Points ».

—. (1994), Condition de l'homme moderne, Paris, Pocket, coll. « L'agora ».

-. (1986), «De l'humanité dans de "sombres temps". Réflexions sur Lessing», dans Vies politiques, traduit de 
l'allemand par Barbara Cassin et Patrick Lévy, Paris, Gallimard, coll. « Tel», p. 11-41.

BeCKETT, Samuel. ([1980], 1993), Company / Compagnie and A Piece of Monologue / Solo. A Bilingual Variorum Edition, ed. by Charles Krance, New York / London, Garland.

Bergounioux, Pierre. (2010). « Hétérogamie », dans Dominique VIART et Jean-Bernard VRAY (dir.), François Bon, éclats de réalité, op. cit., p. 145-149.

—. (1995), La Cécité d'Homère, Strasbourg, Circé.

Bon, François. (2006 [2004]), Daewoo, Paris, Le livre de poche.

—. (1998), Impatience, Paris, Minuit.

—. (2007 [1982]), Sortie d'usine, Paris, Minuit.

CHAUDIER, Stéphane. (2010), "Daewoo, un roman marxiste? », dans Dominique VIART et Jean-Bernard VRAY (dir.), François Bon, éclats de réalité, op. cit., p. 165-175.

Chauvin, André. (2006), "Voix ouvrières dans Mémoires de l'enclave (Jean-Paul Goux) et Daewoo (François Bon)», dans Corinne GrenoulLLet et Éléonore REverzy (dir.), Les voix $d u$ peuple dans la littérature des XIX et XX siècles. Actes du colloque de Strasbourg, Strasbourg, Presses universitaires de Strasbourg, p. 361-377.

ELLMANN, Richard. (1987), James Joyce, II, traduit de l'anglais par André Coeuroy et Marie Tadié, Paris, Gallimard, coll. « Tel »..

Florey, Sonya. (2010), « De Sortie d'usine à Daewoo : chronique d'une mutation», dans Dominique VIART et Jean-Bernard VRAY (dir.), François Bon, éclats de réalité, op. cit., p. 151-163. 
JÉRUSALEM, Christine. (2010), « Tournage, ajustage et fraisage : copeaux de mémoire ouvrière dans Daewoo et Billancourt de François Bon », dans Dominique VIART et Jean-Bernard VRAY (dir.), François Bon, éclats de réalité, op. cit., p. 177-183.

VAN MonTfrans, Manet. (2010), " "Faire face à l'effacement" : voix du réel et voix du théâtre dans Daewoo", dans Dominique VIART et Jean-Bernard VRAY (dir.), François Bon, éclats de réalité, op. cit., p. 185-199.

RANCIÈRE, Jacques. (2010), «Communistes sans communisme? », dans Alain BADIOU et Slavoj ŽIžEK, L'Idée du communisme, Fécamp, Nouvelles Éditions Lignes, p. 231-245.

—. (1995), La Mésentente. Politique et philosophie, Paris, Galilée, coll. « La philosophie en effet ».

VIART, Dominique et Bruno VERCIER avec la collaboration de Franck EVRARD. (2008), La littérature française au présent. Héritage, modernité, mutations, Paris, Bordas.

Villa, Dana R. (2008), Arendt et Heidegger. Le Destin du politique, Paris, Payot, coll. «Critique de la politique».

VRAY, Jean-Bernard. (2010), "Entre bascule et abîme: le franchissement des frontières », dans Dominique VIART et Jean-Bernard VRAY (dir.), François Bon, éclats de réalité, op. cit., p. 129-142.

ŽIžEK, Slavoj. (2008), Fragile absolu. Ou pourquoi l'héritage chrétien vaut-il d'être défendu, Paris, Flammarion.

—. (2007 [2005], Bienvenue dans le désert du réel, traduit de l'anglais par François Théron, Paris, Flammarion, coll. «Champs ». 


\title{
Résumé
}

De ses premiers romans, publiés chez Minuit, aux livres les plus récents, l'œuvre de François Bon a connu une évolution importante. Si les préoccupations politiques n'étaient pas absentes de Sortie d'usine, elles étaient encore classiquement organisées par le registre de l'aliénation des travailleurs, audacieusement figurée par l'héritage formaliste de l'écrivain. Le retour à ce motif de l'usine à travers Daewoo donne à lire une double mutation : celle du politique, de plus en plus insaisissable et pourtant lourd d'effets - à l'image du Réel et celle du récit qui cherche à le figurer. C'est à la mise en scène de micro-relations en tant que figurations du politique à l'état embryonnaire que je m'intéresserai, cherchant à repérer dans cette recension des rapports intersubjectifs une archéologie du politique qui se confond avec la nature même du récit plutôt que d'en être simplement l'objet.

\begin{abstract}
From his first novels, published by Éditions de Minuit, to his most recent books, Bon's work has shown a notable evolution. If political concerns were not lacking in Sortie d'usine, they were still classically organized according to the register of worker's alienation, though daringly figured through the writer's formalist heritage. The return of the factory as a subject in Daewoo reveals a double mutation: that of the political, increasingly elusive yet heavy in its effects - just like the "Real" - and that of the narrative aiming at its figuration. This article will thus focus on the representation of micro-relations as figures of the political in an embryonic state, seeking to locate in this critical revision of intersubjective relations an archaeology of the political that merges with the narrative itself, rather than simply being its object.
\end{abstract}

